\title{
Situational Construction and Information Transmission of Display Atmosphere
}

\author{
Longfei Huang \\ Wuhan Textile University \\ Wuhan, China 430073
}

\begin{abstract}
Based on the characteristics and advantages of modern information environment, this paper analyzes the relationship between the "contextualization" of display atmosphere and the speed and efficiency of information transmission. This paper proposes that exhibition planning and design should be based on the theme and object of the exhibition and set the environment related to its original ecology or significance of existence, which enables the audience to fully mobilize hearing, knowing, smelling, touching and other sensory factors in the specific and real situation to experience and receive the complete information about the exhibition, and then guide the audience to use their own perceptual ability to sublimate passive acceptance into emotional communication and interaction, so as to achieve the best speed and efficiency of information transmission.
\end{abstract}

Keywords-display; atmosphere; contextualization; information transmission; speed and efficiency; efficiency

\section{INTRODUCTION}

The "contextualization" of exhibition atmosphere is a unique expression different from other information media and carriers. Nowadays, with the development of network information, display designers should make full use of the unique advantages of this display form, and give full play to its characteristics of onsite, immediacy, authenticity, interactivity and effectiveness, which enables the audience to be exposed to the most on-site and effective "contextualized" exhibition atmosphere, effectively mobilize their own perception ability, sublimate passive acceptance into emotional communication and interaction, improve the speed and efficiency of information transmission, and achieve the most effective "persuasion" effect.

\section{THE CHARACTERISTICS OF DISPLAY AND} INFORMATION TRANSMISSION IN INFORMATION AGE

Information transmission is one of the important means to promote the progress of human society. The information transmission chain in modern society is composed of information sender, information medium and information signal, information receiver (i.e. the audience). Among them, the information sender has a strong purpose of communication, persuasion, induction and indoctrination, which is an active behavior; while the information source of information receiver, namely "the audience", has the duality. One part is passive acceptance, such as audiovisual advertising, outdoor advertising and public media information with mandatory and diffuse characteristics. The other part comes from the information collection that the "audience" actively performs for some requirements. The former achieves the transmission effect under the influence of persistent information bombing. The latter is more direct and effective with clear purpose. Among them, the most common is visit museum and exhibition.

In today's information age, the Internet is like a highway set up in an invisible space, reaching in all directions, and people can obtain any information they want through online inquiry. The new round of scientific and technological revolution centered on information technology has greatly shortened the space and time distance of each component of the world market, and provided the most convenient means for the development of global trade, investment and financial business. Taking the rise of "e-commerce" in recent years as an example, according to a report released by the United Nations, global e-commerce transactions reached US \$377 billion in 2000 and US $\$ 1$ trillion in 2010. In the next 10 years, one-third of global international trade will be completed in the form of online trade. According to statistics, there are about 87 million Internet users in China at present, and more than $40 \%$ of them have the experience of Internet shopping. In 2003, the market value of online trading reached 4.2 billion Yuan. It can be seen that online trading is becoming increasingly perfect and gradually entering the mainstream of public consumption and enterprise trading, which has brought a great impact on the exhibition industry. But even under this impact, the world exhibition industry still develops steadily. For example, Germany, the world's largest exhibition country, has 23 large-scale exhibition centers with a total exhibition area of 2.5 million square meters and 100,000 exhibition employees, with an annual comprehensive output value of 41 billion Mark. The exhibition industry of the United States, Italy, France, Britain, Japan, Singapore, Hong Kong and other countries and regions has developed greatly in recent years, accounting for a considerable proportion in the national economy of these countries or regions. According to the estimates of the authoritative figures of the international exhibition industry, the output value of the international exhibition industry accounts for about $1 \%$ of the total output value of 30 trillion US dollars. Plus the benefit that relevant industry obtains from exhibition, its output value is quite considerable. Exhibition industry in China is entering a rapid development 
stage, and has become a thriving hot industry. Exhibition planners are among the second batch of new jobs in China released by the Ministry of Labor and Social Security in early December 2004. This benefits from the exhibition's own advantages. Among the information media and communication forms that reside on the transmission chain, the most on-site, intuitive, authentic, interactive and effective one is the exhibition, which is the fundamental reason for the existence and development of the exhibition. It is also the basic value of the exhibition.

Visiting an exhibition is an active behavior. Visitors come to the exhibition for face-to-face contact, communication, trade negotiation, collection and acquisition of information, etc. The purpose and communication atmosphere are much simpler than the direct transaction. As a result, visitors are in a lightly guarded state of mind where communication is much easier. The face-to-face, two-way, direct and intuitive information exchange environment provided by the exhibition site is suitable for high-quality information exchange and superior to one-way, indirect and passive communication. Therefore, it has a profound appeal and a wide range of mass, and is easy to achieve the expected social publicity and guidance, education role.

The development of the Internet in the information age cannot replace the real exhibition, but is conducive to the development of the exhibition industry. Online exhibition (virtual exhibition form) should be a useful supplement to physical exhibition, and modern information means become a powerful tool to display, opening up a new look for modern exhibition activities. At the same time, the objectivity, authenticity and trustworthiness of the exhibition are irreplaceable. Due to the appearance of "virtual display", the display in the information age will pay more attention to the "contextualization" of the display site to achieve emotional resonance and communication with the audience.

\section{INFORMATION DISSEMINATION FUNCTION, CHARACTERISTICS AND "CONTEXTUALIZATION" CONSTRUCTION OF THE EXHIBITION}

For the purpose of information transmission, promotion, education and enlightenment, exhibition is a visual communication method or phenomenon that effectively transmits the intended contents to visitors within a certain limited time or specific space. As an information medium and a unique form of communication, exhibition expo covers specific space and time environments such as fairs, museums, art galleries, shop windows and display cases, shopping malls, temporary celebration venues, etc. Its meaning is to show or emphasize what is unknown to others or to reinterpret what is known. The main concept and goal of exhibition planning and design is clear and convincing demonstration.

This form of information exchange has the characteristics that other forms of information exchange do not have: materiality, immediacy and centrality. Exhibition can produce direct "eyeball effect" through its "eventfulness" and "sense of on-site", and improve its intrinsic potential of added value, which is the value of information transmission on the basis of exhibition. The image of the "exhibit" in the exhibition itself is different from its image in other media. The real purpose of visitors to the exhibition site to obtain information is the content of internal information and intuitive meaning contained in the exhibits. For the exhibitor, that is, the information exporter, when choosing a scheme to successfully improve the rate and efficiency of information transmission in the best way, it is necessary to create a display environment corresponding to the exhibits through the creation of "situational" atmosphere, so as to help the audience get an intuitive feeling of "immersive", so as to achieve the purpose of information transmission. This should be the most effective form of persuasion.

Clavde Shannon and Weaver pointed out that there are three problems in the communication research: first, technical problem. It is necessary to consider how to convey the transmission symbol accurately. The second is semantic problem. It is necessary to consider whether the transmitted symbol can convey the message correctly. The third is the efficiency problem. It is necessary to consider whether the received message can effectively influence and guide the receiver to achieve the desired goal. The effectiveness in the third layer is done by the sender and the receiver. The efficiency of information transmission comes from persuasion, and the success of persuasion depends on the emotional mobilization of the "audience". After receiving the information, the "audience" will synthesize its own inherent information and store it together to form emotions such as "happy, angry, sad, happy, love and hate", and form the "decoding" of the information, so as to directly affect the judgment and selection of the information.

The information transmission function of "display" is mainly completed by means of nonverbal communication. The intended information is communicated with the audience through the display environment composed of various elements such as target group, theme, media and space. The modern display environment should be a multi-faceted, omni-directional, multi-media three-dimensional environment space. "Contextualization" construction means that in the process of exhibition planning and design, scientific methods, theories, experiments, concepts, procedures and other aspects related to exhibition must emphasize "contextualization". According to the theme and object of the exhibition, people should set up an environment related to its original ecology or meaning of existence, so that the audience can fully mobilize hearing, perception, smell, touch and other sensory factors in the specific and real situation to experience and receive complete information about the exhibition, guide and activate the audience's own perception ability, elevate the passive acceptance into emotional communication and interaction, so as to impress the audience and sublimate the value of the exhibits and the exhibition in the specific situation. Finally, the optimal speed and efficiency of information transmission are achieved to complete the whole process of information transmission.

"Contextualized" exhibition can bring a kind of "experience", which is to give visitors a kind of spiritual shock, give them joy, and experience something they have never experienced before coming to visit. And "experience" 
is valuable. People spend money on travel, art shows, museums and so on to seek an "experience". The development of experience value is currently lacking in commercial exhibitions. However, it is a key to transform from ordinary exhibition to brand exhibition and from basic value to added value. If an exhibition can bring visitors a strong experience, it will form a "polarization" and "magnetization" effect in the minds of visitors. If this effect is strong enough, it may solidify into a "concept". The exhibition that can bring experience to the visitors must have a distinct theme, vivid situation and unique style, and it must be able to stimulate the visitors from senses to feelings to thinking, and finally form action.

Exhibitions are planned for established purposes. Depending on the theme, the presentation will be presented in different forms, styles and situations. Take "auto show" as an example. The North American International Auto Show in Detroit is one of the top five auto shows in the world. In recent years, concept cars have made up a growing share of North American auto shows. Every year, more than 40 automobile manufacturers and over 700 new concept and production vehicles participate in the exhibition, which is an important showcase for the global automobile industry. The 2005 North American International Auto Show in Detroit featured dynamic features, new materials, and exquisite production to highlight the show's consistent, highly technological and civilized "concept cars". In particular, Chrysler's new Jeep Hurricane off-road vehicle, introduced in 2005, was so good that it hung the Jeep Hurricane booth on a man-made cliff. This indicates the striking forward and backward angular characteristics of the Hurricane. (See "Fig. 1") The Hurricane is "almost vertical in and out of rough terrain, making climbing a wall seems like an easy task for the Hurricane". Cars are called "toys for grownups" and are often compared to "beautiful cars" for successful people. In Europe and the United States, the SUV is a portrayal of male heroism. The extraordinary ability of the new model is reflected and rendered through the real "situation", which can also inspire the trust of fans and put it into action. Thus, the purpose of information communication of the auto show has been perfectly realized.

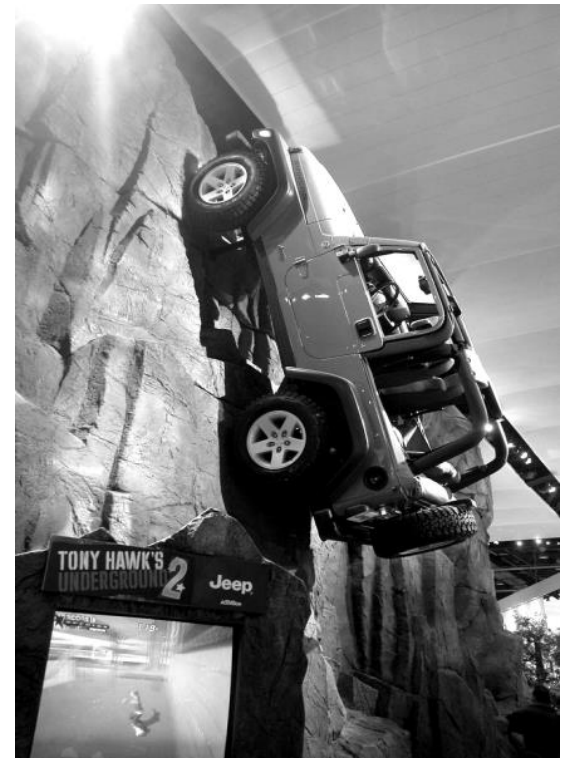

Fig. 1. Jeep Hurricane booth.

The 2005 E3 game show in Los Angeles was a different story from the full-color auto show aimed at adults: the intense impact of fiery colors, exaggerated images and visual continuance from the outside to the inside shows the audience preferences of the exhibition, as well as the youth, commotion and fashion characteristics of the game industry, which makes the gamers and fans have a desire to try and understand new game products. (See "Fig. 2")

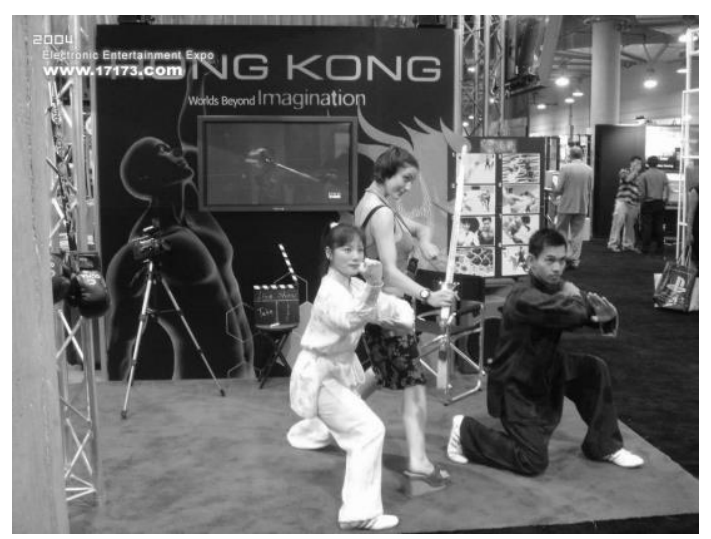

Fig. 2. The 2005 E3 game show in Los Angeles.

\section{CONCLUSION}

Thus it can be seen that an exhibition can establish, advocate and spread its own "soul" through the "contextualization" of the exhibition atmosphere. In this way, the inner information connotation of the exhibits and the spiritual concept of the information sender can realize the transmission of in-depth information to the audience, rather than the visual transmission of superficial meaning, so as to improve the speed and efficiency of information transmission of the exhibition activities. 


\section{REFERENCES}

[1] Zhang Ming. Creative China Pavilion [J]. China Conference \& Exhibition. 2003. 6. (in Chinese)

[2] [US] Zeert, translated by Zhao Sensen. Sight Sound Motion: Applied Media Aesthetics [M]. Communication University of China Press, 2003, 10, 1st edition. (in Chinese)

[3] Liang Wen. Economic Globalization and International Exhibition Industry [N]. Economic Daily. 2002-07-17. (in Chinese)

[4] Yang Weidong. Present Situation and Development Tendence of Chinese Electronic Business Affairs [J]. China Agricultural Information Bulletin. No. 4, 2001. (in Chinese) 